\title{
A RARE CASE OF RIGHT SIDED UNILATERAL DYSMENORRHOEA
}

Jamila Hameed ${ }^{1}$, Narmada ${ }^{2}$, Sathyavani ${ }^{3}$

\section{HOW TO CITE THIS ARTICLE:}

Jamila Hameed, Narmada, Sathyavani. "A Rare case of Right Sided Unilateral Dysmenorrhea". Journal of Evolution of Medical and Dental Sciences 2014; Vol. 3, Issue 17, April 28; Page: 4556-4559,

DOI: $10.14260 /$ jemds/2014/2474

ABSTRACT: Most of the adolescent girls complain of pain during periods. The incidence of dysmenorrhea is $25 \%$. A 17 year old girl came with right sided pain which was mistaken for appendicitis and had laparoscopic appendicectomy. She was referred to gynecology outpatient department since the pain on the right side was not relieved even on medical treatment. Repeatedly she took leave from her school. At one stage patient was thought to be malingering and was referred for psychotherapy. MRI showed class IV bicornuate unicollis uterus, with partially underdeveloped rudimentary horn with functional endometrium. The provisional diagnosis prior to laparotomy was bicornuate uterus but on surgery it was found to be a unicornuate uterus with non-communicating rudimentary horn on the right side with functioning Endometrium. There was no associated renal abnormality and endometriosis. The functioning unicornuate horn was attached to the uterus by a fibrous band which was excised easily. The patient was relieved of dysmenorrhea. In unilateral dysmenorrhea the probability of congenital malformation should be thought of. Early diagnosis and management will help to prevent a lot of complications like endometriosis, infertility, ectopic pregnancy and rupture of underdeveloped pregnant horn.

CASE REPORT: A17 year old girl was referred from surgical department who had laparoscopic appendicectomy for right sided lower abdominal pain. She had severe dysmenorrhea for the past 3 years. The pain was typically felt over the right lower abdomen and it started just before the menstrual cycle, reached its peak on the third day of the menstrual cycle. She also gave a history of taking analgesics on and off for it. On examination, her vitals were stable and no abnormality was detected, everything was normal except for the tenderness at the right iliac fossa. She was advised ultrasonography (USG) to rule out any pelvic pathology. USG showed bicornus unicollis rudimentary horn 35 x 31mm with endometrium. (Figure 1) To confirm the diagnosis, magnetic resonance imaging (MRI) was performed showing class - IV bicornuate unicollis uterus, relatively small sized or partially underdeveloped rudimentary horn with functional endometrium and right hematometra. (Figure 2) Intravenous pyelography showed no renal pathology. Even though laparoscopy is preferred we did laparotomy.

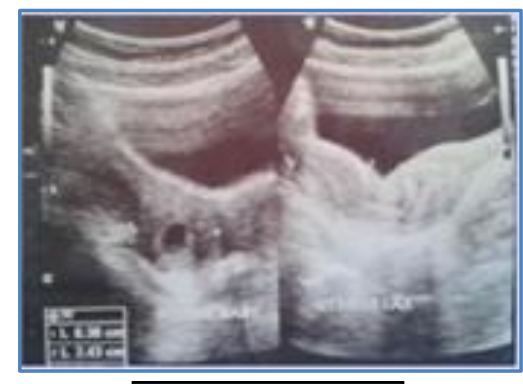

Fig. 1: (USG)

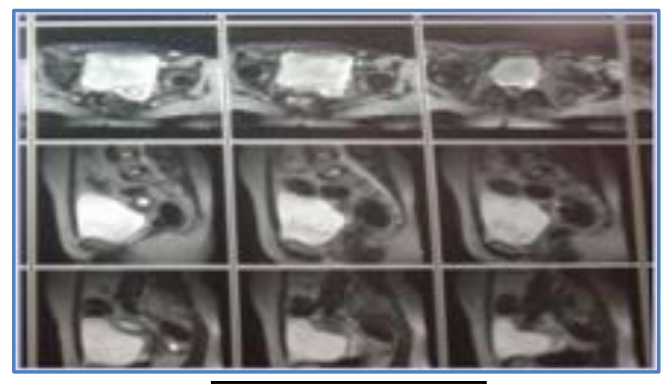

Fig. 2: (MRI) 
On laparotomy, we found the functioning left sided uterus had a rudimentary horn of size $3.5 \times 3.3 \mathrm{~cm}$ on the right side which was found attached by a fibrous band (figure 3). The right rudimentary horn had no communication to the left side uterus which had proper cervix although it had functional endometrium. The fallopian tube was absent on right side. On the left side, the fallopian tube was found to be normal (figure 4). Both the ovaries were normal. On excising the rightsided horn of the uterus, sticky chocolate like material came out, establishing the diagnosis of hematometra. The excision was easy by cutting through the fibrous band without entering into the uterine cavity, and maintaining proper hemostasis. The excised specimen was then sent for histopathological examination confirming the diagnosis of rudimentary horn with myometrial and endometrial tissue. Her post-operative period was uneventful. The patient had attended our outpatient department for follow-up and had no complaint of dysmenorrhea. She happily attended the school without any absence.

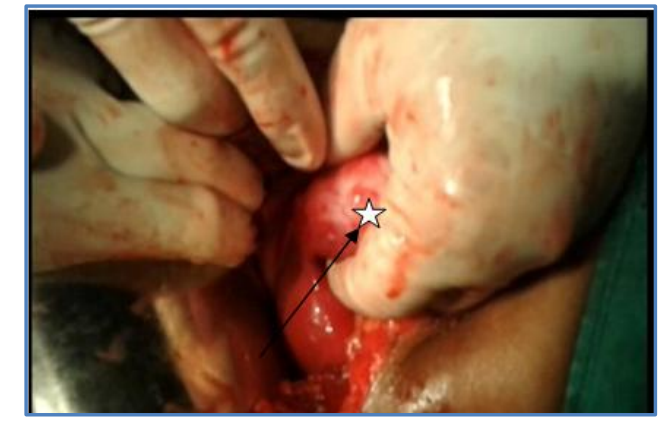

Fig. 3: Right sided rudimentary horn

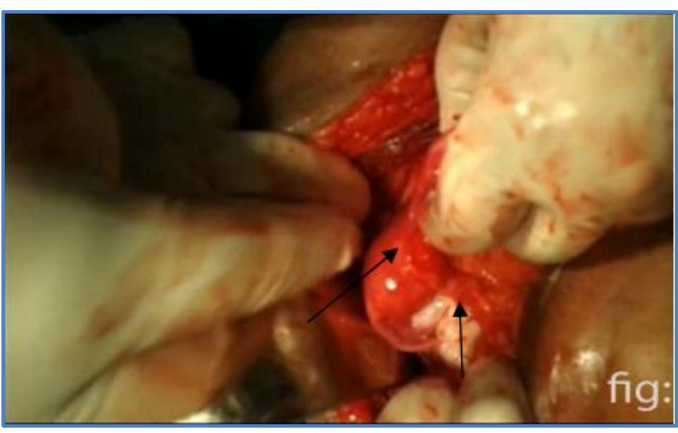

Fig. 3: Left sided uteruswith ovary

DISCUSSION: Unicornuate uterus with a rudimentary horn is a rare type of mullerian duct malformation, with an incidence of 1 in 100000. ${ }^{1}$ The rudimentary horn may consist of a functional endometrial cavity or it may be a small solid lump of uterine muscle with no functional endometrium. According to American Society 1998 mullerian anomalies were divided into 4 groups. ${ }^{2}$ This case belongs to II A-1-b in which the rudimentary horn is non-communicating with the opposite site horn. Mullerian anomalies are commonly associated with gross malformation of the renal tract, spinal and cloacal anomalies. In our case she had severe unilateral pain continued on the right side even after appendicectomy and other causes were ruled out like renal colic, myoma and endometriosis, the probability of uterine abnormality was thought of. When there was a doubt regarding uterine abnormalities, the following investigations like three dimensional USG, ${ }^{3}$ MRI which is the gold standard ${ }^{4}$ were helpful. Hysteroscopic intervention should be used if the rudimentary horn is communicating with the uterine cavity. Here in this case the presence of fibrous band prevented the myometrial injury 5 because there is no need to enter the uterine cavity. The removal of the fallopian tube on the right side in order to prevent ectopic pregnancy ${ }^{6}$ was not needed as it was absent in our case. The provisional diagnosis prior to surgery by scan was bicornuate uterus but on surgery it was found to be a unicornuate uterus with non-communicating functioning rudimentary horn. When a woman presents with unicornuate uterus there is an increased incidence of infertility, endometriosis, dysmenorrhea and the obstetrical complications like spontaneous abortion, ectopic pregnancy, rupture of the rudimentary horn, preterm deliveries, caesarean deliveries and maternal mortality ${ }^{7}$. A 
unicornuate uterus causes few symptoms and it is usually discovered by chance or as a result of pregnancy complications. But, in our case, the patient presented with unilateral dysmenorrhea. The dysmenorrhea in this case was due to the obstruction to the outflow of menstrual blood in the functioning rudimentary horn. Other causes of unilateral dysmenorrhea may be endometriosis with unilateral distribution or a small leiomyoma at the uterotubal junction. One-sided spasmodic dysmenorrhea in a young girl should always raise the suspicion of uterine malformation, and every effort should be made to exclude this condition by conducting relevant investigations. The diagnosis of mullerian abnormalities can be made by USG. However, MRI is more specific for the evaluation of presence or absence of a functional endometrium. But, the gold standard to diagnose mullerian anomaly is diagnostic laparoscopy.

It is generally considered that the presence of a non-communicating rudimentary horn carries increased risks of endometriosis and cornual pregnancy. To avoid such serious complications in the future, early diagnosis and excision of the rudimentary horn is of great significance. Laparoscopic excision is ideal to the young girls as it is more acceptable due to cosmetic reasons.

CONCLUSION: Unilateral dysmenorrhea is a not routinely seen in general gynecological practice and usually presents as acute abdomen. It should be investigated in an adolescent girl presenting with dysmenorrhea with relevant investigations keeping in mind the rare possibility of mullerian duct anomalies. Early diagnosis and management of this condition is essential to prevent to avoid gynecological and obstetrical complications like hematometra, hematosalpinx, endometriosis, ectopic pregnancy and torsion. Timely intervention with the maximal suspicion of this abnormality and minimal access surgery is recommended. Unilateral dysmenorrhea due to functional non communicating horn was relieved by its excision.

\section{REFERENCES:}

1. Atmaca R, Germen AT, Burak F, Kafkasli A. Acute abdomen in a case with non-communicating rudimentary horn and uni-cornuate uterus. JSLS 2005 Apr-Jun;9(2):235-7.

2. Buttram VC Jr, Gibbons WE. Müllerian anomalies: a proposed classification. (An analysis of 144 cases). Fertil Steril. 1979 Jul; 32(1):40-6.

3. Salim R, Woelfer B, Backos M, Regan L, Jurkovic D. Reproducibility of three-dimensional ultrasound diagnosis of congenital uterine anomalies. Ultrasound Obstet Gynecol. 2003 Jun;21(6):578-82.

4. Troiano R.N. Magnetic resonance imaging of mullerian duct anomalies of the uterus. Top Magn Reson Imaging. 2003 Aug; 14(4):269-79.

5. Perrotin F, Bertrand J, Body G. Laparoscopic surgery of unicornuate uterus with rudimentary uterine horn. Hum Reprod. 1999 Apr;14(4):931-3.

6. Handa Y, et al. Tubal pregnancy in a unicornuate uterus with rudimentary horn: a case report. Fertil Steril. 1999 Aug;72(2):354-6.

7. Heinonen P.K. Clinical implications of the unicornuate uterus with rudimentary horn. Int J Gynaecol Obstet. 1983 Apr;21(2):145-50. 


\section{CASE REPORT}

\section{AUTHORS:}

1. Jamila Hameed

2. Narmada

3. Sathyavani

\section{PARTICULARS OF CONTRIBUTORS:}

1. Professor, Department of Obstetrics \& Gynaecology, Vinayaka Mission's Medical College \& Hospital, Karaikal.

2. Tutor, Department of Obstetrics \& Gynaecology, Vinayaka Mission's Medical College \& Hospital, Karaikal.

3. Post Graduate, Department of Obstetrics \& Gynaecology, Vinayaka Mission's Medical College \& Hospital, Karaikal.

\section{NAME ADDRESS EMAIL ID OF THE} CORRESPONDING AUTHOR:

Dr. Jamila Hameed,

Professor,

Department of Obstetrics \& Gynaecology,

Vinayaka Mission's Medical College \& Hospital, Karaikal, Pondicherry (UT) - 609609.

E-mail: jamilahameed@gmail.com

Date of Submission: 26/03/2014.

Date of Peer Review: 27/03/2014.

Date of Acceptance: 08/04/2014.

Date of Publishing: 25/04/2014. 